\title{
Editorial
}

\section{Function Spaces, Compact Operators, and Their Applications}

\author{
S. A. Mohiuddine, ${ }^{1}$ M. Mursaleen, ${ }^{2}$ Adem Kiliçman, ${ }^{3}$ and Abdullah Alotaibi ${ }^{1}$ \\ ${ }^{1}$ Department of Mathematics, Faculty of Science, King Abdulaziz University, P.O. Box 80203, Jeddah 21589, Saudi Arabia \\ ${ }^{2}$ Department of Mathematics, Aligarh Muslim University, Aligarh 202 002, India \\ ${ }^{3}$ Department of Mathematics and Institute for Mathematical Research, University Putra Malaysia, 43400 Serdang, Selangor, Malaysia
}

Correspondence should be addressed to S. A. Mohiuddine; mohiuddine@gmail.com

Received 7 July 2014; Accepted 7 July 2014; Published 14 September 2014

Copyright (C) 2014 S. A. Mohiuddine et al. This is an open access article distributed under the Creative Commons Attribution License, which permits unrestricted use, distribution, and reproduction in any medium, provided the original work is properly cited.

The aim of this special issue is to focus on the latest developments and achievements of the theory of compact operators on function spaces and their applications in differential, functional, and integral equations. The concept of the compactness plays a fundamental role in creating the basis of several investigations conducted in nonlinear analysis and is very useful in several topics of applied mathematics, engineering, mathematical physics, numerical analysis, and so on. The compactness is very often used in fixed point theory and its applications to the theories of functional, differential, and integral equations of various types. On the other hand, the sequence spaces offer relevant tools for illustrating abstract results and properties in functional analysis.

The research papers in this special issue cover various topics like function spaces and compact operators on them, sequence spaces and their topological and geometric properties, paranormed Nörlund sequence spaces of nonabsolute type, spaces of functions over the field of non-Newtonian complex numbers, statistical summability methods and their application to Fourier series, applications of Hankel and regular matrices in Fourier series, statistical approximation results for Kantorovich-type operators, Mellin transform and Kratzel transform, fixed point theory and its applications, Fourier transform, convergence methods of iterative algorithm, isomorphic universality, Sobolev type spaces, fractional integral operators, well-posedness and stability for Euler equations, integral equation-wavelet collocation method, functional differential equations, Urysohn integral equation, difference equations, and Lipschitz spaces and integral operators.

\section{Acknowledgments}

The editors thank all the contributors and colleagues who did the refereeing work very sincerely.
S. A. Mohiuddine M. Mursaleen Adem Kiliçman Abdullah Alotaibi 


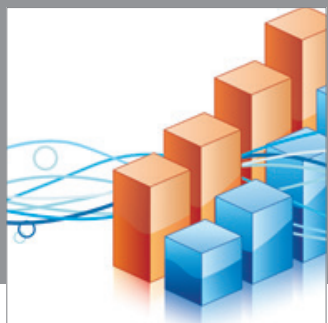

Advances in

Operations Research

mansans

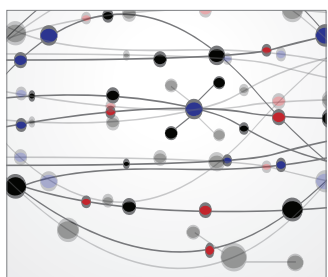

The Scientific World Journal
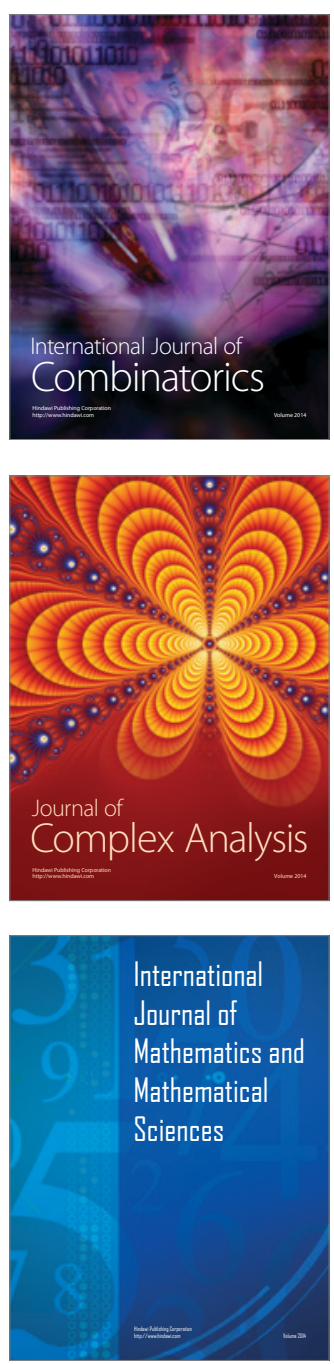
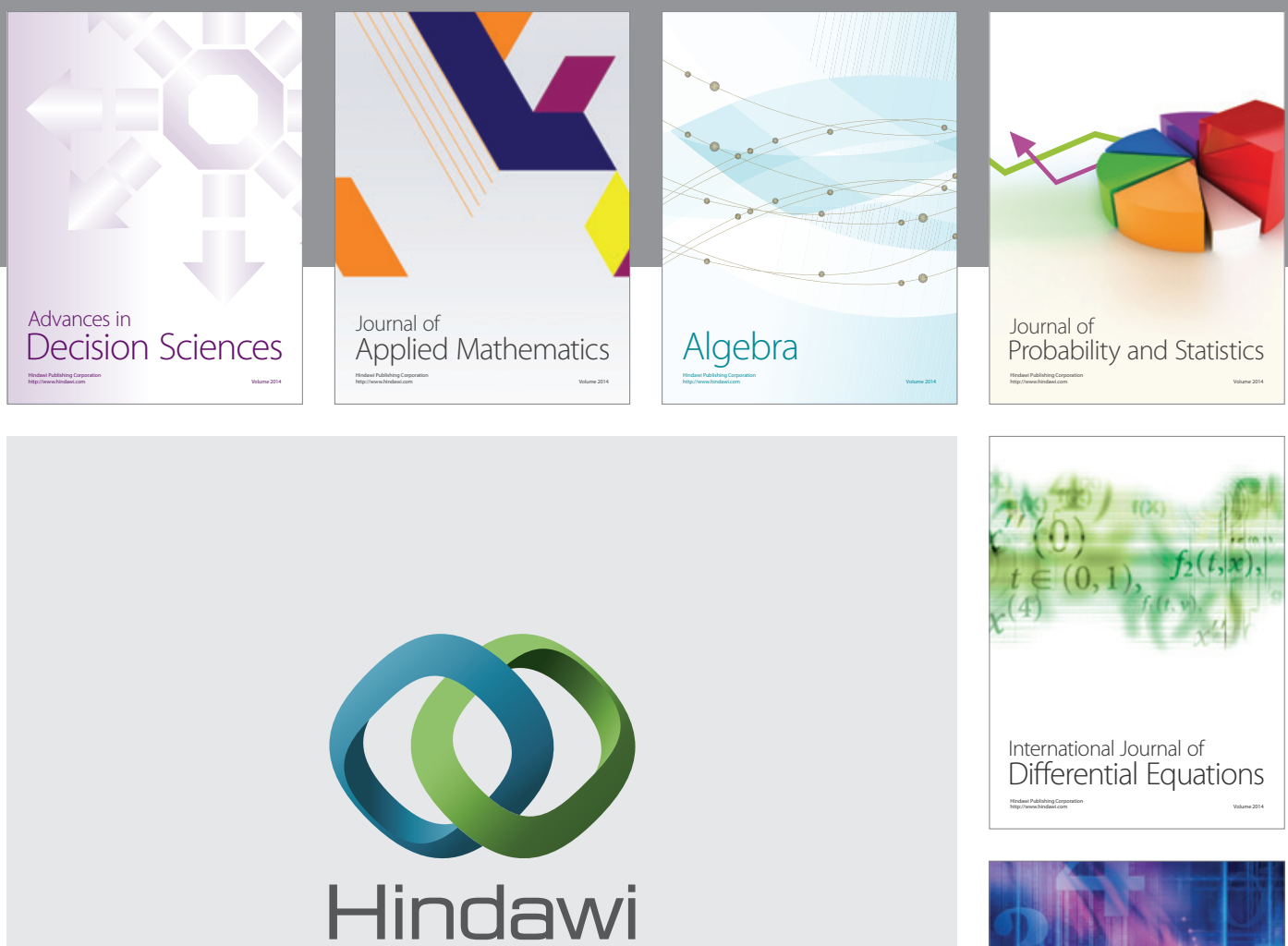

Submit your manuscripts at http://www.hindawi.com
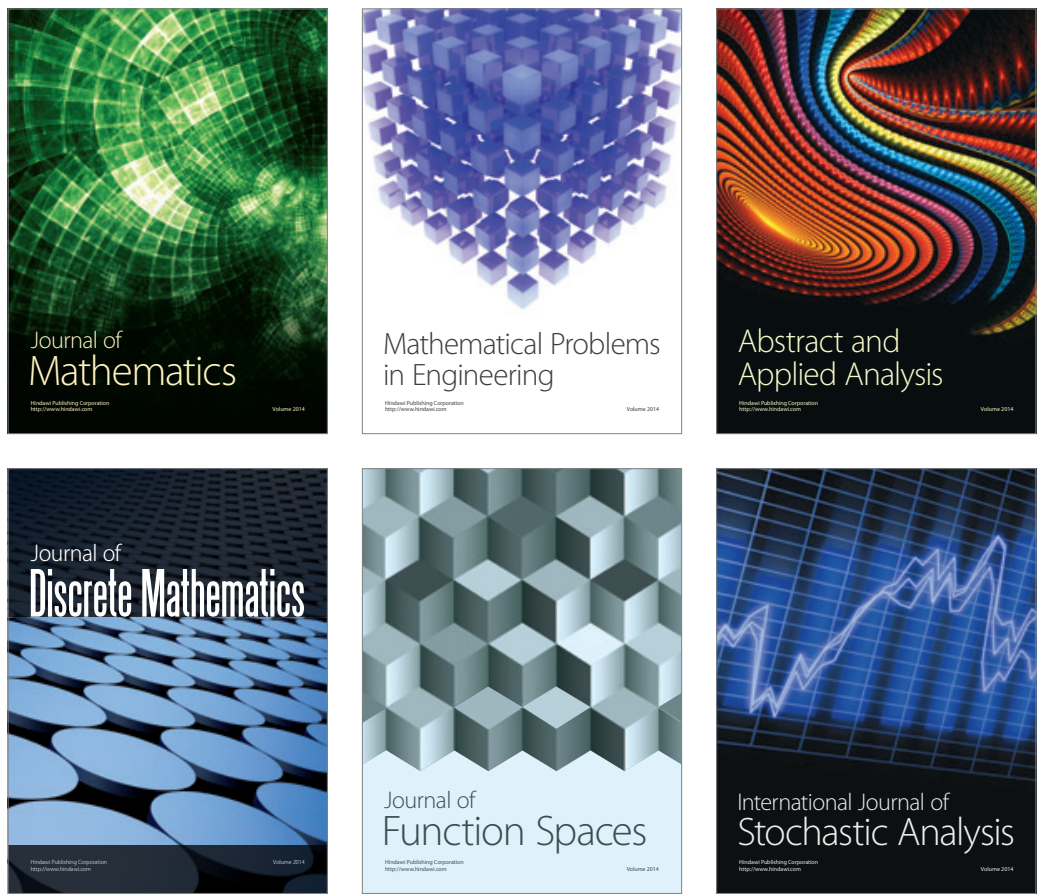

Journal of

Function Spaces

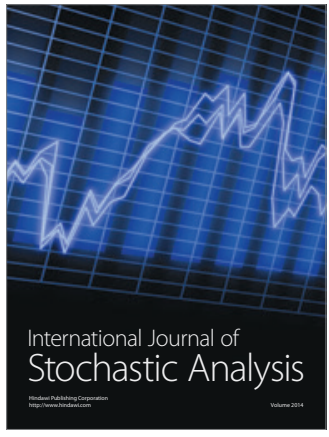

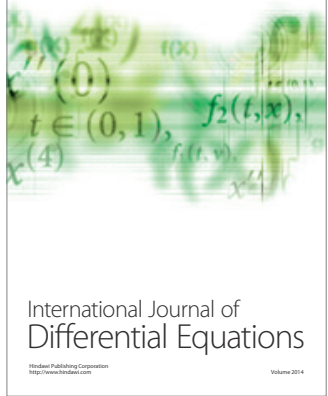
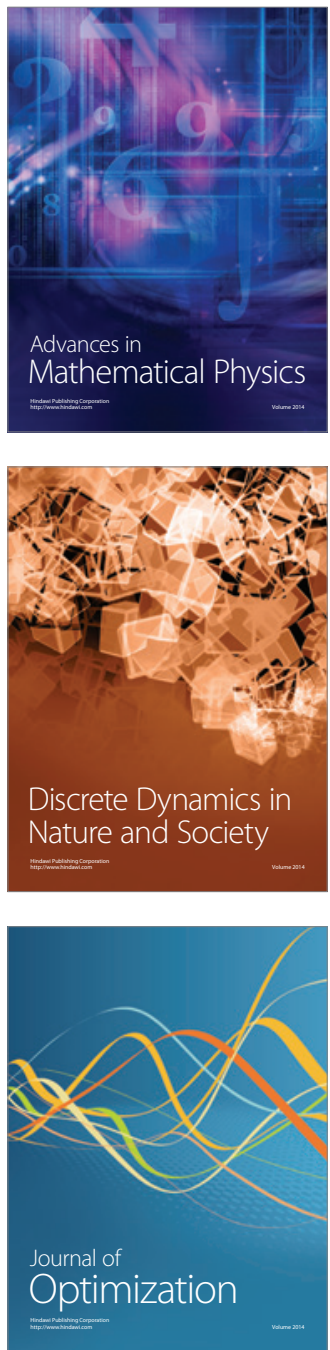